\title{
ORgANIZATIONAL FACTORS IN DETERMINING DATA QuAlity PRODUCED FROM HEALTH MANAGEMENT INFORMATION SYSTEMS IN LOW- AND MIDDLE-INCOME COUNTRIES: A SYSTEMATIC REVIEW
}

\author{
Thein Hlaing ${ }^{1}$ and Thant Zin $^{2}$ \\ ${ }^{1}$ District Public Health Department (Ministry of Health and Sports), \\ Pyay District, Bago Region, Myanmar \\ ${ }^{2}$ Faculty of Health and Social Sciences, STI Myanmar University, Yangon, Myanmar
}

\begin{abstract}
The Health Management Information System (HMIS) is an essential core component in framing the national health system. To operate six core components synchronically and to manage them successfully inside the health system, HMIS and communication are also placed centrally. However, the unworthy problems of HMIS data have been significantly affected by several characteristics. Among these characteristics, the organizational factors need to be considered as important issues. This systematic review aims to examine what organizational factors are determining the HMIS data quality in LMICs after 2005. Two independent reviewers selected 38 eligible primary published papers from 22 LMICs through three popular online sources: MEDLINE and PubMed, HINARI, and Google and Google Scholar. This finding mainly highlighted that weak organizational structuring and processing, less organizational learning development regarding HMIS, unavailability of HMIS resources, poor governance, and political issues impacted the HMIS data quality in LMICs.
\end{abstract}

\section{KEYWORDS}

HMIS data quality, Organizational determinants \& LMICs

\section{INTRODUCTION}

The health system is framed with six core components such as provision of healthcare services, health manpower, health management information systems (HMIS), equitable access to treatment with essential medicines, financial saving, and leadership and governance ${ }^{1}$. To operate these components synchronically and to manage them successfully inside the health system, HMIS and communication are placed centrally ${ }^{2}$. Importantly, every HMIS has been corresponding for the highest availability and accessibility of sound and accurate health information ${ }^{3}$. The data delivered from HMIS are recognized as the foundation for system decision-makers to track system performance and progress of health status, to analyze the health-related impacts, and to ensure accountability within the health system ${ }^{4}$. Further, the HMIS data are vital to analyze what health problems are present, to indicate what actions are needed, and to support superior and central decision-makers for their sound effective decisions for the improvement of the health system $^{5,6}$. 
The important information about almost all healthcare programmes such as maternal and child health, adolescent health, elderly health, other specific disease prevention, and control programmes and so on is carried out through the HMIS regularly and collectively ${ }^{7,8}$. Thus, the importance of the HMIS data for human health starts from the first hour of their lives when they are deeply sleeping inside their mothers' wombs to the last minute of their lives ${ }^{8}$. The data resulting from well-operated HMIS are essential for formulating and implementing health system policies, undertaking further researches, strategically managing human resources, services, and finances, governing and regulating the health system, and evaluating the target achievements ${ }^{1}$. Also, these data are capable of early warning health status and determining health situations and trends. Most health plans and programmes are established according to the basics of these data ${ }^{1,9}$, 10 .

However, the unworthy problems of HMIS data have been significantly affected by several characteristics. Among these characteristics, the organizational structure, culture, climate, processes, and policies need to be considered as the important issues because these determine the smoothness of workflows, the exercise of certain powers, the availability of the skilled staff, supports, supervisions, leadership and governance concerned with the HMIS ${ }^{11}$. The organizational structure is the framework for achieving common organizational goals, in which the workforce is managed through certain rules, roles, and responsibilities. Structuring the organization needs to be efficient and effective because it determines the flows of information and decision-making power. In the HMIS field, the organizational structure needs to be considered as an important issue. It is also crucial for organizing relationships and defining responsibilities to make the effective arrangement of HMIS staff and to operate the HMIS tasks cooperatively together ${ }^{11}$. The comprehending of how appropriately the structure of an organization is set up, how liquidly individuals communicate through an organization structure designed, and how effectively individuals within it perform HMIS is a critical issue for managing the HMIS data production.

The collective efforts of the HMIS users through rules, values, practices, roles, and responsibilities also impact the HMIS data production process ${ }^{12}$. Watkins ${ }^{13}$ depicted that organizational culture influences behavioural patterns. Similarly, the organizational culture determines the HMIS performance and has the direct and indirect effects on the HMIS data quality through values, beliefs, attitudes, and perceptions of the HMIS users ${ }^{12}$. Besides, to maintain the quality culture and the best performance of HMIS, it is also important to understand how organizational processes are managed. Many organizational processes such as interrelation, network creation, information exchange, flexible and delayering operation, communications, team building, and their behaviours, levels of engagement, power delegations, administrative manners and ability to lead are considered as factors affecting the HMIS performanc ${ }^{7,12}$.

Moreover, organizational climate concerns with perceptions of the HMIS users towards their working environment and it depends on the different individual value judgments within the organization and also affects motivation, behaviours, performances, and productivities of the HMIS users ${ }^{12}$. Additionally, the inefficacy of a culture of information decreases the HMIS performance. High quality in the measurement of the culture of information relates to the highquality status of HMIS data, the effective utilization of HMIS information, the ability to decide with evidence basis, the capability of addressing the problems, and better responsibility and accountability of the HMIS staff ${ }^{14}$. Therefore, the HMIS needs to be harmonic with the organizational characteristics and in other words, the HMIS deviated from the original communication flow of the organization is not easy to be the best performance ${ }^{15}$. However, the organizational characteristics come to be questioned how completely and adequately these are structured and operated for keeping the good performance of the HMIS in the form of dynamic function. Currently, redesigning and strengthening national HMIS of LMICs is vital, for which a 
clearer rethinking of what organizational problems exist in the data production process of HMIS and which organizational factors are challenging the HMIS data quality is essential.

Moreover, many HMIS authorities from LMICs who need reliable evidence-based information for HMIS reform are difficult to adapt to the up-to-date situation due to poor technological adaptation and limited time and resources. Further, they are unwieldy evidence supports from massive numbers of published papers with diverse languages from different countries. Besides, the organizational factors influencing on the data quality of HMIS in LMICs may be different across the countries ${ }^{16,17}$. To be usable and glaringly obvious, these factors from disparate studies need to be aggregated into a single collaborative form. This study is interesting to do a systematic review for reacting to such challenges and aims to examine what organizational factors are determining the HMIS data quality in LMICs after 2005. Hopefully, this baseline evidence will be a complete and exhaustive summary for HMIS developers, users, and implementers in LMICs to monitor and tackle the improvement of HMIS data quality.

This review paper is structured with five sections such as the introduction of the study, research methodology, results, discussion, and conclusions. The introduction section explains the basic information of HMIS, the importance of HMIS data in LMICs, and how the organizational factors associated with the HMIS data quality, why this review should be done, and the objectives of this review. The second section covers a detailed presentation of the research methodology and explains how this systematic review was conducted. The result section describes the detailed selection process with words and flow diagram (Figure.1) and characteristics of the included studies with words, figure.2, and table. 1 as well as summarizes the main findings according to the organizational theme and sub-headings. The fourth section discusses the significant findings and their particular differences from other previous reviews that focused on the HMIS fields. This section also discusses how this review finding is different or similar to the existing evidence-base and previous studies, explains how this review has strengths and limitations and presents the reviewers' expectation for future work. In conclusion, main findings and the contributions of the review are briefly summarized.

\section{RESEARCH METHODOLOGY}

\subsection{Research Design}

The research design employed was a systematic review because it can result in more complete and exhaustive evidence than those from a primary study ${ }^{18}$. This design will be a better design to profitably support the collective experiences from many LMICs for better planning and implementing HMIS.

\subsection{Formulation of Research Question}

This study's research question was "what determinants exist in the quality data production process of HMIS in LMICs after 2005?" The reviewers formed this question according to the PICOT framework. In this question, HMIS data quality was the problem $(\mathrm{P})$ and the HMIS data production processes were interventions or exposures (I). The reviewers interested the organizational determinant factors of HMIS data quality as the outcome $(\mathrm{O})$ and considered the events after 2005 (Time frame $(\mathrm{T})$ ). However, this review formulated the research question without consideration of comparison (C) because a PICOT analysis does not always address this portion $^{19}$. 


\subsection{Eligibility Criteria}

This review determined the eligibility criteria on the relevance and acceptability basis for including the studies that meet the predetermined research question and predefined research objective.

\subsubsection{Types of Studies}

The sample of studies used in this review considered the quantitative, qualitative, and mixed methods. The rationale for considering mixed-method studies is to gather the outcomes resulted from their synergistic data collection and analysis, to compare the results from qualitative or quantitative methods only, and to try reaching more complete conclusions. As this review included the studies conducted in many LMICs, this inclusion will provide an alternative answer for a defined research question. This review tacked together the studies reported in English. The rationale for excluding non-English studies, because there will be a particular difficulty of translation for reviewers, which will lead to well-interpretation without translation errors. This review was more likely to assemble the studies published in 2005 or after. This inclusion was rationalized because, since 2005, LMICs have been introduced to pilot or deploy DHIS2. Additionally, this review tried to identify and to foregather the published studies in peer-reviewed or scholarly journal articles or high-quality papers because the accuracy, quality, and usefulness of the contents of peer-reviewed journal articles have already been examined by the particular expert team before publication. Only primary studies were considered in doing this review.

\subsubsection{Eligible healthcare settings and participants}

The research papers eligible for this review have to study the organizational determinants of the HMIS performance or the HMIS data production processes in the primary, secondary and tertiary healthcare settings separately or serially. As well, the studies studying on the participants like the HMIS technicians (e.g. software developers), the HMIS users (e.g. the HMIS focal persons, nurses, statisticians, managers, coordinators, officers, and supervisors of the HMIS), the HMIS donors and agencies were eligible for this review.

\subsubsection{Eligible intervention}

This review related to the studies focused on the performance of the HMIS or the processes of HMIS at any level. For example, data registration/recordation, entry into reports, compilation, reporting, processing, analyzing, dissemination, and other related activities like system management and monitoring processes that intend to make certain of the data quality were recognized as eligible interventions. Also, the included studies concerned with the components of HMIS namely health management information system, health information system, electronic record system, district health information system software-2 (DHIS-2), the hospital record system, the hospital management information system and the hospital information management system, the routine health information system and the routine public health information system.

\subsubsection{Outcome measures}

The organizational determinant factors affecting the data quality production processes or performance of the HMIS at any level in LMICs were expected as outcome measures. 


\subsection{Bibliographic Searching}

Three major online sources such as MEDLINE and PubMed, HINARI, and Google and Google Scholar, were used for each eligible study. As the supplementary approaches, the reviewers searched carefully the potentially includable papers from other websites such as regulatory agency websites, WHO and related websites and checked enthusiastically the reference lists as well as contacted the publishers and authors for being more complete search. To limit or permit the nature of all related studies to the predefined research question, the most typical terms or keywords were used as search terms. Additionally, the search strategies were certain by using Boolean Operators (OR and AND), Proximity Searching, Generalized Search, Focused Search, Double Quotation Marks, Limiters (e.g. Date range, Language, Types of studies) and Expanders. For being potentially relevant for this review, two reviewers worked together for searching independently the pertinent full-text articles according to the list of core search terms such as country names, organizational problems, factors, influencers, determinants, barriers, obstacles, different terms of HMIS, different indicators of HMIS data quality, etc.

\subsection{Identification of the Primary Researches}

Screening the typical titles and abstracts of the primary researches were done by two reviewers because undertaking a review by a single reviewer should be avoided and error reduction may be resulted from doing a review by two or more independent reviewers. After searching and retrieving independently, each reviewer downloaded and saved all relevant articles in the EndNote programme that was applied for avoiding duplications of the studies, and then noted how many studies were identified from each database. When there was a duplication of selected studies between reviewers, the reviewers counted the total number of relevant studies by removing duplicated studies.

\subsection{Data Extraction}

For objectively criticizing, presenting, and summarizing the relevant evidence from a core of studies, the reviewers developed the data extraction sheet and pre-tested it on ten randomly selected papers of a final set of included studies, and refined it accordingly for being consistent, reliable, and standardized sheet. Furthermore, all definitions of variables in this spreadsheet were specified. Additionally, the reviewers cross-checked all data extracted.

\subsection{Critical Appraisal of Studies}

The quality of methodological domains of pertinent studies was assessed independently by two reviewers applying detailed explanations of the Mixed Methods Appraisal Tool (MMAT), version 2018 developed by Pluye et $a l^{20}$. The overall quality score of each retained study was computed using the MMAT and scored as $20 \%\left({ }^{*}\right)$ when one criterion of the relevant component of the MMAT met, $40 \%(* *)$ when two criteria met, $60 \%(* * *)$ when three criteria met, $80 \%(* * * *)$ when four criteria met and $100 \%(* * * * *)$ when all criteria met.

\subsection{Ethical Consideration}

This review was approved by The Ethics Committee of the School for Healthcare Practice, the UK on $6^{\text {th }}$ March 2020. The reviewer tried hard not to be a misleading summary of this review and this summarized evidence will inform according to available information included in the published papers. Besides, the reviewer's reflections will guarantee the anonymity of the origins of information and these will be in the range of unit guidelines. 


\subsection{Data Analysis}

In this process, all eligible full-text articles were printed out and cut according to their subheadings (e.g. Methodology, Findings) and then piled according to their similar sub-headings, but every portion cut was ensured by attaching with a paper that included the title of the article, publication year and name of authors. After that, the main findings were extracted and synthesized narratively on the representative themes of organizational determinants. The extracted data were descriptively analyzed rather than quantitatively. The reviewer evaluated and reported the between-study variability by categorizing or logically combining individual study outcome measures to provide bigger evidence representing all included studies.

\subsection{Research Duration}

After this review has been approved on $6^{\text {th }}$ March 2020, all review processes started in the second week of March 2020 and this review was successfully completed on 12 June 2020.

\section{RESUltS}

\subsection{Summary of Selection Process}

A total of 5836 titles and abstracts (2961 from reviewer-1 and 2875 from reviewer-2) were initially identified through three main databases. Additionally, 41 relevant published papers $(22$ from reviewer-1 and 19 from reviewer-2) were searched and retrieved from other data sources (see Figure.1). Finally, 38 papers with 3747 participants were selected for this present review.

\subsection{Characteristics of Included Studies}

Of the 38 papers, $13(34.21 \%)$ were quantitative studies, 11(28.95\%) were qualitative studies and $14(36.84 \%)$ were mix-method studies. Of these, 19(50\%) concentrated on the primary healthcare settings' HMIS, $8(21.05 \%)$ studied on the primary and secondary healthcare settings' HMIS, 4(10.53\%) concentrated on secondary healthcare settings' HMIS, 2(5.26\%) focused on secondary and tertiary healthcare settings' HMIS and 5(13.16\%) studied all possible determinants factors of HMIS data quality at all levels. 
Health Informatics - An International Journal (HIIJ) Vol.9, No.4, November 2020

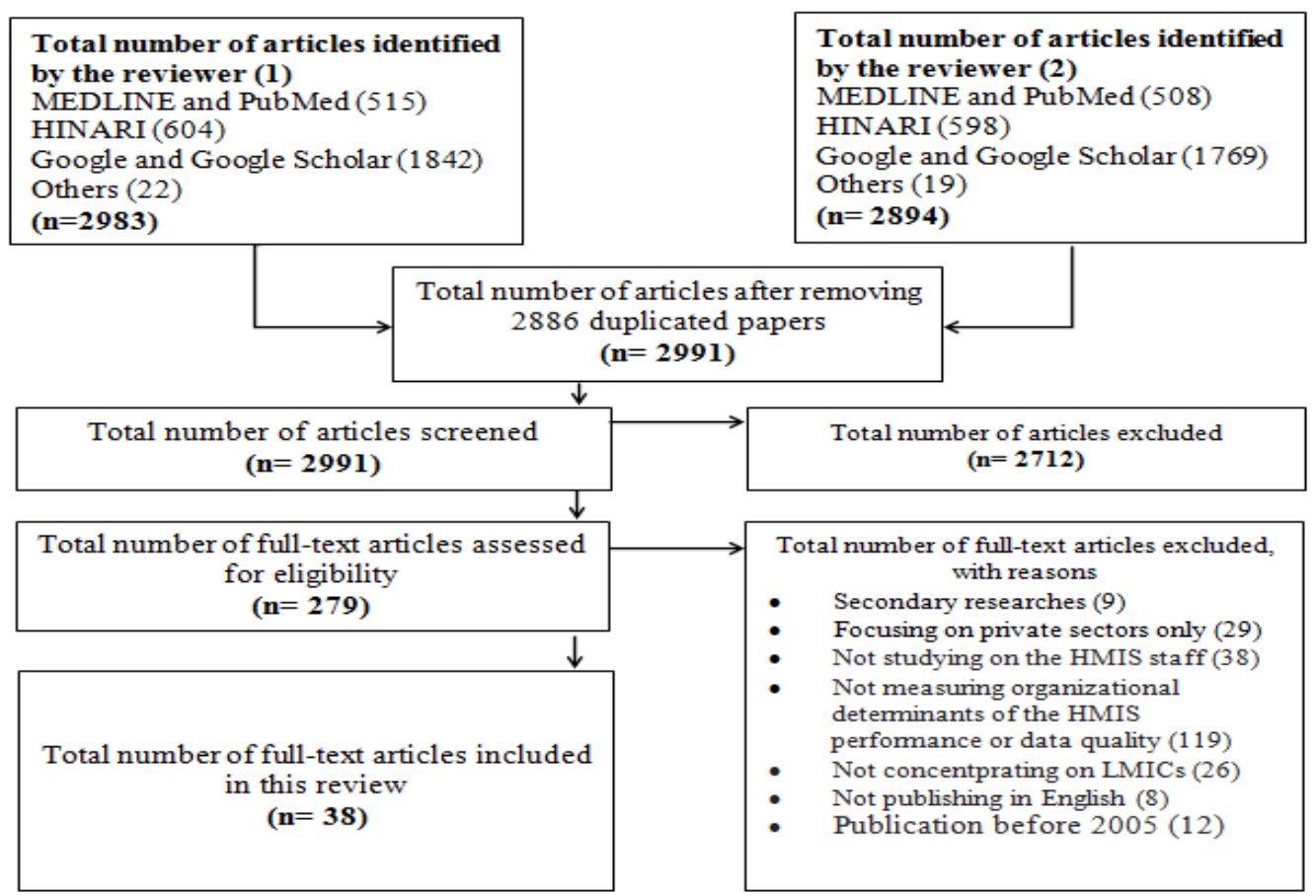

Figure.1. Flow diagram showing the study selection processes

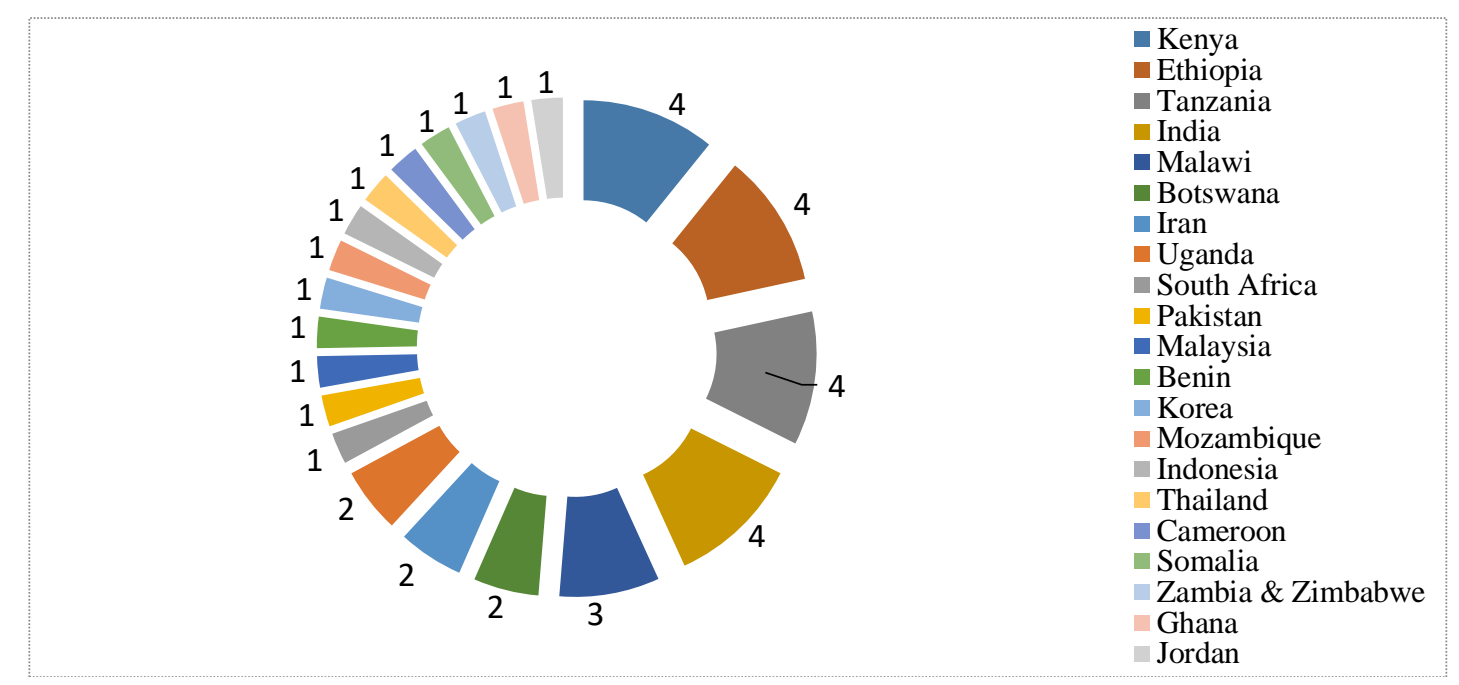

Figure.2. Composition of the included studies by LMICs

The vast majority $(89.5 \%)$ of the pertinent studies was undertaken by employing the crosssectional descriptive study designs and a smaller number of studies employed the cross-sectional analytical design (2.62\%), the interventional design $(5.26 \%)$, and longitudinal design $(2.62 \%)$. Of the selected studies that investigated factors determining the performance or data quality of the HMIS, 34(89.47\%) measured all possible determinant factors, 3(7.89\%) highlighted to find out only organizational factors, and other 1(2.64\%), identified both behavioural and organizational factors. The selected studies were conducted in 22 LMICs (see Figure.2).

The included studies were published between 2006 and 2019 and six studies produced their outcomes through case studies of Tanzania ${ }^{21}$, India ${ }^{22}$, Somalia ${ }^{23}$, Cameroon ${ }^{24}$, Indonesia ${ }^{25}$, and 
Jordan $^{26}$. The included studies concerned with a broad range of study participants and applied different types of data collection tools and methods (see Table.1).

Most papers in this review studied the determinant factors by directly focusing on the HMIS data quality or performance ${ }^{16,22,24,25,27-43}$. Some papers studied the HMIS to explore their gaps and challenges encountered ${ }^{26,44-48}$, to evaluate the strengths and weaknesses of HMIS functional components $^{23,49,50}$, to analyze the management and evaluation portions of HMIS ${ }^{51}$, to assess the consolidation of the HMIS data and associated factors ${ }^{52}$, to examine the challenges and facilitating factors of HMIS on the basic of the data quality of the disease-specific programmes such as TB, HIV/AIDS and Malaria ${ }^{21}$, and maternal health programme ${ }^{53}$, to explore the determinants of the e-HMIS's sustainability ${ }^{54,55}$ and to evaluate the DHIS2's adoption ${ }^{56,57}$.

After appraising the methodological qualities of the selected studies using MMAT (Version 2018), papers in this review had a quality score ranging from $40 \%$ to $100 \%$. Of total selected studies, $31(81.58 \%)$ had a high-quality score (80\% and above) because of: (1) their appropriate approach for research problems, (2) random, clear and adequate sample selection procedures, (3) delineated samples, (4) relatedness of the target population, (5) preliminary testing of questionnaires' validity and reliability, (6) adequate data collection methods, (7) low nonresponse rate, (8) appropriate statistical analysis and adequate findings and (9) clear links between data sources, processing and interpretation. 7 out of the selected studies $(18.42 \%)$ had a quality score by $60 \%$ and below, for this to happen, the non-probability sampling procedure, unclear description of inclusion and exclusion criteria, unclear explanation of reasons for conducting the mixed-method studies, disconnection of the quantitative and qualitative data analysis and interpretation were the most prominent reasons.

Table.1.Identifications of study participants and data collection tools of the included studies
(1) HMIS
Technical partners, IT staff, Software developers,
technicians
Software advisors, and Software managers
(2) HMIS users
HMIS in-charges, HMIS focal persons, Health directors, Medical specialists, Environmental health officers, Medical officer-in-charge, Doctors, Assistant medical officers, Lab technicians, Pharmacists, Nutritionists, Counselors, Immunizers, Nurses, Midwives, Lady health volunteers, Health facility management staff, Ward clerks and Statisticians

\section{Identifications \\ of study \\ participants}

\section{(3) HMIS programme managers}

(4) Heads of school

(5) HMIS donors (6) Others
HMIS programme/data managers and coordinators, M\&E officers, Information officers and HMIS supervisors

Headmasters of nursing schools and University professors

HMIS donors and Support agencies

Social health activists 
Health Informatics - An International Journal (HIIJ) Vol.9, No.4, November 2020

\begin{tabular}{|c|c|c|}
\hline & (1) Quantitative data & $\begin{array}{l}\text { Structured questionnaires } \\
\text { Semi-structured questionnaires } \\
\text { Interviewed/self-administered questionnaires } \\
\text { the PRISM tools } \\
\text { Data Quality Assurance tools } \\
\text { Data quality review tools } \\
\text { Document reviews } \\
\text { Assessments of interventional effects }\end{array}$ \\
\hline $\begin{array}{l}\text { Data } \\
\text { collection } \\
\text { tools/ methods }\end{array}$ & (2) Qualitative data & $\begin{array}{l}\text { Key In-depth Interview (KII) } \\
\text { Focus Group Discussion (FGD) } \\
\text { Interviewing with semi-structured questionnaires } \\
\text { Interviewing with structured } \\
\text { Interviewing with opened-end questionnaires } \\
\text { Observations } \\
\text { Document reviews } \\
\text { Multi-stakeholder workshops } \\
\text { Discussions }\end{array}$ \\
\hline
\end{tabular}

\subsection{Findings of the Study}

\section{Organizational Determinants}

For implementing HMIS successfully, the organizational structure and function are very important to fit in HMIS goals and missions. Thus the organizational aspects are viewed as the topmost inputs of HMIS because certainties in organizational aspects can ensure the HMIS policies, guidelines, plans, resources, and other important management strategies that are necessities of good quality data of HMIS. This review identified that several organizational uncertainties and challenges affected the HMIS data quality in LMICs.

\subsubsection{Organizational Structure}

This review identified that the organizational structure was one issue in importance to the HMIS data quality improvement. The most common organizational determinant was the absence of the HMIS focal person who had a responsibility to upper-level authorities for the HMIS tasks ${ }^{16,21,23 \text {, }}$ 35, 40, 41, 43, 50, 52. Simba and Mwangu ${ }^{40}$ found that the HMIS data completion rate was about 1.5 times higher in health facilities with HMIS focal person than in health facilities without. Also, a higher completeness rate of the HMIS data was more likely to be found among the in-charges who have a responsibility to the district authorities for the HMIS activities than those without. Another important organizational determinant was the lack of definite roles and responsibilities of the HMIS implementers ${ }^{50,51}$ and hence, they lacked job security ${ }^{51}$. Locations and general features of healthcare settings, their organic managerial structures ${ }^{23}, 24,55$, the HMIS administrator's ability to manage the HMIS tasks ${ }^{24}, 32$, job category and education level of the HMIS staff ${ }^{27}$ and their burdensome works and work pressure ${ }^{36,37}$ needed to be considered as other additional organizational issues for higher performance of HMIS.

\subsubsection{Organizational Processes}

This review also identified that poor process of the organization affected the HMIS performance. Poor quality of the HMIS data resulted from poor communication in the HMIS workflow. For instance, the HMIS officers at national levels missed disseminating their well-developed HMIS 
indicators to district levels timely to health facility levels absolutely ${ }^{50}$. Besides, the HMIS administrators lacked discussion or evaluation meetings about the HMIS performance regularly ${ }^{30}$, 36, 41, 43, 48. Further, the HMIS developers and users processed HMIS activities without cooperation, collaboration, and networking ${ }^{35,52}$. These poor processes of organization lead to poor organizational culture. As a result, the HMIS produced duplicated, inconsistent and unmanageable data ${ }^{42,44,57}$.

\subsubsection{Organizational Learning}

Knowledge, understanding, and insights about the importance of the HMIS were found as big issues determining the data quality. Regarding organizational learning about HMIS, operating the HMIS tasks without training or with insufficient knowledge reduced data quality. The HMIS staff learned about HMIS through only a short course or workshop or on-job training that did not cover all contents of the HMIS ${ }^{16,22,24,27,29,30,31,33,34,36,37,38,39,41,43,46,50,52,57}$. Further, the HMIS staff had less opportunity to learn about HMIS-related knowledge due to their insufficient time, work pressure and over-workload ${ }^{27,28,31,44,46,52}$. Additionally, lack of supportive supervision, regular data auditing and positive feedback from upper levels caused less chance for progress in HMIS knowledge and lead to poor HMIS performance ${ }^{22,24,28,30,31,34,42,43,51,52,55}$. Teklegiorgis et $a l .{ }^{42}$ found that good quality data of HMIS was statistically associated with the HMIS staff who possessed good knowledge and skill in performing the HMIS tasks \{COR=3.260, $95 \% \mathrm{CI}(1.742,6.103)\}$.

\subsubsection{Resources Availability}

The studies from Benin, Somalia, Malawi, Ethiopia, Tanzania, and Uganda revealed that the HMIS data quality was importantly determined by HMIS resources availability ${ }^{21,23,27,31,52}$. The HMIS data quality was directly affected by the shortage of health manpower, inadequate HMIS staff, insufficient supply, and distribution of HMIS tools and infrastructure $e^{21,22,24,35,36,37,41,45,57}$. Moreover, the very limited national budget for performing HMIS functions, for purchasing and maintaining the HMIS equipment and infrastructure, for documenting HMIS records and for training HMIS staff also directly affected the HMIS data quality ${ }^{29,41,43,52}$. Additionally, running out of stock on essential health products had an indirect effect on the HMIS data quality ${ }^{43}$.

\subsubsection{Governance and Political Factors}

Inefficient processes and poor performance of the HMIS were directly linked with great deals about governance. In performing the HMIS activities, management without a fair political framework, management by personal point of views of organizational politicians, inappropriate management of the HMIS staff's recruitment, turnover, and their workplaces, less accountability of the HMIS staff, lack of shared decision makings, inability to maintain compliance of organizational culture and inability to increase staff's participation were important challenges to the HMIS performance improvement ${ }^{31,44,52}$. Also, the poor HMIS performance was associated with the unclear and unfair allocation of the national budget in the HMIS activities ${ }^{26,37,38,41,54}$. Further, lack of performance reviews on HMIS ${ }^{30,31,52}$ and lack of management support from upper-level HMIS staff ${ }^{57}$ were other issues affecting the HMIS performance. Moreover, the HMIS processes and performance proceeded without standardized policies, guidelines and strategic planning for how to compile the HMIS data, how to practice cross-checking and data transmission $^{52}$, how to maintain documents, how to protect confidentiality $23,26,29,50$ and how to upgrade IT infrastructure ${ }^{42}$. Additionally, the staff's transfer without proper handover of the HMIS documents was another important issue to be considered as a political determinant of the HMIS performance ${ }^{35}$. 


\section{Discussion}

This review aimed to identify all possible organizational factors in determining HMIS data quality in LMICs after 2005. To meet this aim, the exhaustive searching of the eligible papers published in 2005 and after was performed. However, the finding covers the determinant factors that emerged in 2006 and after.

This review is particularly different from the previous reviews. Specifically, this review studied the HMIS developers, donors, and users responsible for the HMIS data production processes or performance, and focused on many health information systems as well as reviewed primary papers published in 2006-2019. Whereas, a review of Akhlaq et al. ${ }^{58}$ studied the participants responsible for HMIS information exchange, focused on only electronic HMIS, and considered unpublished and published papers conducted in 2009-2014. Further, the reviews of Mohadali and Aziz ${ }^{59}$ and Mohadali and Aziz ${ }^{60}$ that aimed to determine the organizational and technical barriers of the HMIS studied on papers conducted in Malaysia and published in 2010 and after. Their reviews could not cover the experiences of other LMICs apart from Malaysia. Furthermore, the reviews of Garavand et al. ${ }^{61}$ and Ross et al. ${ }^{62}$ determined the applicability, impacts, and user perceptions of using e-HMIS and their entire attention focused on the technology portion of the HMIS. Garavand et al. ${ }^{61}$ reviewed the systematic reviews published in 2004-2014 while Ross et $a l .{ }^{62}$ reviewed the systematic, narrative reviews and qualitative meta-synthesis published in 20092014. Then a literature review of Sezgin and Yildirim ${ }^{63}$ studied the quantitative primary researches published in 2002-2012 and determined m-Health technology users' attitudes towards the implementation of e-health and m-health information systems. The reviews of Garavand et $a l .{ }^{61}$, Ross et al. ${ }^{62}$, and Sezgin and Yildirim ${ }^{63}$ were typical for LMICs. Also, a systematic review and meta-analysis of Lua et al. ${ }^{64}$ focused on systematic review articles conducted in high-income countries and published in 1996-2008 as well as aimed to investigate the HMIS effects on the healthcare quality.

Under the theme of the organizational determinants, this review identified that lack of enough HMIS staff or responsible person for the HMIS tasks is the major characteristic to determine the HMIS data quality. This characteristic is consistently found through many papers included in this review. Like these included papers conducted by Asah and $\mathrm{Saebo}^{24}$. Husain, Saikia, and Bora ${ }^{22}$, Kasambara et al. ${ }^{36}$, Kpobi, Kumwenda et al. ${ }^{37}$, Ledikwe et $a .^{50}$, Simba and Mwangu ${ }^{40}$, Swartz, and Ofori-Atta ${ }^{45}$, Tadesse, Gebeye, and Tadesse ${ }^{41}$, Teklegiorgis et al. ${ }^{42}$, Wagenaar et al. ${ }^{43}$ and Wandera et $a .^{52}$, a study from Northwest Ethiopia on the use of the HMIS data coherently considered the shortage of responsible professionals in performing HMIS as a big organizational issue $e^{65}$. Then this review identified that limited training programme for HMIS capacity development is another unchanged organizational characteristic in LMICs' HMIS. This characteristic is seen in several papers conducted by Cheburet and Odhiambo-Otieno ${ }^{29}$, Dehury and Chatterjee ${ }^{53}$, Farzandipur, Jeddi, and $\mathrm{Azimi}^{32}$, Husain, Saikia, and Bora ${ }^{22}$, Ismail et al. ${ }^{49}$, Kasambara et al. ${ }^{36}$, Kpobi, Swartz, and Ofori-Atta ${ }^{45}$, Kumwenda et al. ${ }^{37}$, Ledikwe et al. ${ }^{50}$, Malik and Hameed ${ }^{38}$, Manya and Nielsen ${ }^{16}$, Mishra et al. ${ }^{39}$, Tadesse, Gebeye, and Tadesse ${ }^{41}$, and shows that the HMIS administrators from LMICs still need to develop and implement a more proactive and strategic plan for the HMIS staff's capacity building. Similarly, Lua et al. ${ }^{64}$ reported that the development activities for HMIS knowledge and skill are primarily regarded as fundamentals of effective management and performance of HMIS. Other studies correspondingly reported that less understanding and inability in HMIS tasks are root causes of poor management and low quality of HMIS data and providing typical HMIS training is an important issue to promote the HMIS users' satisfaction and to implement the HMIS effectively ${ }^{62,63,66}$.

This review found other important organizational issues that the HMIS staff in LMICs has no work specialization and job security and then they operate the HMIS tasks with insufficient time 
and resources and poor organizational culture. Moreover, they manage the HMIS tasks without standardized policies, guidelines, and plans. Consequently, the HMIS data quality is poor. In comparing these issues with the review results of Lua et al. ${ }^{64}$ during 1996-2008, equal experiences are also demonstrated, but these issues might be superannuated for high-income countries while they are stated as current HMIS problems in LMICs. Other studies like this review identically reported that lack of basic principles and guidelines, inappropriate division of HMIS staff and tasks, and negative workplace culture are the bad influences on how specifically to perform HMIS ${ }^{67,68}$. Besides, providing the technical, monetary and infrastructure resources less than minimum requirements is the key organizational challenge of maximized HMIS data quality ${ }^{61}{ }^{69}$. Regarding the HMIS policy planning guidelines, the finding of Ross et al. ${ }^{62}$ also stated that the HMIS performance is hampered due to unspecific HMIS legislations, policies and liabilities, and lack of a strategic plan for HMIS. In literature, the organizational culture was depicted as an important factor that influences on behavioural patterns ${ }^{70}$. This statement is supported by this review that the exposure to workplace stressors, supervision-related stressors, workload-related stressors, and lack of incentive cause negative attitudes towards HMIS and in turn produce untimely and inconsistent HMIS data. Thus, comfortable organizational culture and climate, task-shifting, supportive supervision activities instead of practicing only fault-finding and financial motivation are necessary organizational issues for improving positive attitude towards HMIS performance ${ }^{71-76}$.

Additionally, this review found that there are other considerable organizational problems relating to investment, monitoring and evaluation, communication, and workforce management in the HMIS implementation in LMICs. Regarding these problems, other studies harmoniously reported that low investment in HMIS-related activities, lack of regular monitoring and evaluating on the HMIS performance, lack of interaction between the HMIS users, technicians and donors, lack of motivational activities (e.g. performance reviews and reward system development) and improper management of HMIS staff turnover and handover of the HMIS documents are remarkable organizational challenges to the improvement of the HMIS ${ }^{58,59,65}$. In other seven studies conducted in Malawi, Tanzania, Peru, and South Africa, the performance of evaluation meeting focused on the HMIS data quality improvement and formal auditing the HMIS data quality are the big opportunities to understand the obstacles in implementing HMIS and to promote the HMIS data quality by removing these obstacles and providing regular feedback ${ }^{69,77-82}$.

\subsection{Strengths and Limitations}

Although this review is an academic requirement, the employment of a co-reviewer was performed. Using the pilot-tested data extraction sheet reduced bias and mistakes during the collection and extraction of data. Further, to assess the risk of the bias in each study, the review applied the version 2018 of MMAT. Furthermore, a formal reliability exercise, cross-checking, a discussion between two reviewers, and contacting authors and manufacturers resolved the disagreements in selecting the studies, appraising the quality and extracting the data. Besides, as several organizational characteristics of the HMIS data production processes experienced in many LMICs were collectively identified, the overall findings of this review may increase the awareness of these determinants for reforming HMIS in LMICs. Additionally, this review could summarize the perspectives and experiences of a wide spectrum of the HMIS implementers, supervisors, managers, officers, policymakers, technicians, partners, and donors from government and non-government organizations in the HMIS field.

As with other systematic reviews, this review was limited by the accessibility of literature because this review afforded the literature searches regarding peer-reviewed or qualified journal articles that appeared only on online sources. Due to this reason, this review might be a selective or incomplete conclusion on the specific outcome factors that affect the LMICs' HMIS 
performance. Besides, the exclusion of non-English language literature and unpublished materials might threaten the validity of this conclusion and might lead to misrepresentation. Then this review studied many papers with different study periods and publication years and also investigated their possible organizational factors in determining data quality of HMIS within the wide timeframe (2006-2019). This fact might impact the overall result of this review because some determinants concluded in this review might have already been eliminated in some LMICs.

\subsection{Future Work}

According to the prismatic framework, assessment of HMIS performance needs to integrally consider two core portions such as the provision of quality data and utilization of health information ${ }^{9}$. As the factors determining the efficiency and effectiveness of each portion may be slightly different, the complete assessment of all possible factors affecting these two portions can give more visible answers necessary for strengthening the HMIS performance. To meet such requirements, further reviews will be undertaken to investigate the determinants relating to technological and behavioural issues of the HMIS data quality and the determinant factors or barriers in the utilization of HMIS data in LMICs. Also, this review highlighted how the HMIS data quality was determined by several organizational factors, but this could not interest the consequences of poor or good quality of the HMIS data. Especially, after this review has been completed, the research or review will follow to examine the impact of poor quality data from HMIS on healthcare system performance and health status in LMICs. Furthermore, many studies including this review were more likely to highlight challenges, obstacles, and barriers, influencing factors, and determinants of the HMIS performance and less likely to find opportunities for the HMIS performance improvement. Hence, further review that focuses on the identification of several existing opportunities for strengthening HMIS in LMICs will be expected.

\section{Conclusions}

Under the healthcare system, HMIS is mainly responsible for managing healthcare data and for disseminating these data completely, accurately, and timely. Importantly, HMIS is a chief and cost-effective data source for healthcare system management in LMICs. The finding of this systematic review mainly highlighted that weak organizational structuring and processing, less organizational learning development regarding HMIS, unavailability of HMIS resources, poor governance and political issues impacted the HMIS data quality in LMICs. At present, LMICs speedily attempts to achieve universal health coverage and to stand with the action of accomplishing global goals, for which they need timely and accurate health information from HMIS, their main data source. Thus, this review's summary that comes from international experiences contributed to useable reference for developing policies, strategies, and systems to establish a safer, cost-effective, and well-functioning HMIS that can produce the quality data they need. Also, this evidence will guide national health authorities and the HMIS officers from LMICs on how to manage their limited resources effectively by prioritizing the necessary points pinpointed by this review. This review found that there was no smoothness in performing the HMIS tasks due to leadership and government-related problems, strategy, and investment-related problems and resources-related problems. This review contributed to applicable evidence for developing a system-level HMIS strategic plan that covers detailed actions, budgeting plans, human resources, and HMIS knowledge management plan, HMIS infrastructural development plan and plan for networking. Also, this review found that lack of HMIS policy, less interconnectivity between health information systems, and lack of HMIS staff's job security are the barriers in performing HMIS. This finding will push the HMIS authorities in LMICs to develop a national HMIS policy that exactly states data security policy, data confidentiality policy, information exchange and use policy, interoperability standards, and the HMIS staff's roles, responsibilities and job descriptions. Overall, as this review looked at the processes of 
HMIS data production, the HMIS users from LMICs will be easy to see an overview of the organizational determinants that existed in these processes. Managing from the conclusions will help HMIS policymakers, investors, developers, and managers in LMICs to bridge the organizational gaps in quality data production processes.

\section{REFERENCES}

[1] World Health Organization (WHO) (2010) Monitoring the building blocks of health systems: A handbook of indicators and their measurement strategies. Geneva, Switzerland: WHO Press.

[2] MEASURE Evaluation (2013) Health Management Information System (HMIS) Facilitator's Guide for Training of Trainers. Available at: https://www.measureevaluation.org/resources/publications/ms-13-74(Accessed: 11 February 2020).

[3] Orosco, J. (2019) What is a health information system? Available at: https://www.sansorohealth.com/what-is-a-health-information-system/ (Accessed: 9 February 2020).

[4] Lazarus, J.V. (2014) A new era for WHO health system building blocks? Available at: https://www.healthsystemsglobal.org/blog/9/A-new-era-for-the-WHO-health-system-buildingblocks- .html (Accessed: 2 February 2020).

[5] World Health Organization (2006) Framework and Standards for the Development of Country Health Information Systems. Geneva: Health Metrix Network.

[6] World Health Organization (WHO) (2008a) Framework and standards for country health information systems. 2nd edn. Geneva, Switzerland: the Health MetricsNetwork and the World Health Organization.

[7] MEASURE Evaluation (2017) Strengthening health information systems in low-and middle-income countries: A model to frame what we know and what we need to learn. Available at: https://www.measureevaluation.org/resources/publications/tr-17-156. (Accessed: 11 February 2020).

[8] Tan, J. (2010) Health Management Information Systems: A Managerial Perspective. Available at: https://books.google.com.mm/books?id=39Ao9c8blMcC\&pg=PR43\&source=gbs_toc_r\&cad=3\#v=0 nepage\&q\&f=false(Accessed: 11 February 2020).

[9] Mutale, W. et al. (2013) ' Improving health information systems for decision making across five subSaharan African countries: Implementation strategies from the African Health Initiative', $B M C$ Health Services Research, 13(2), doi: 10.1186/1472-6963-13-S2-S9

[10] World Health Organization (WHO) (2008b)Toolkit on monitoring health systems strengthening Health Information System. Available at: http://www.who.int/healthinfo/statistics/toolkit_hss/EN_PDF_Toolkit_HSS_InformationSystems.pdf( Accessed: 5 February 2020).

[11] Kenton, W. (2019) Organizational Structure. Available at: https://www.investopedia.com/terms/o/organizational-structure.asp(Accessed: 9 February 2020).

[12] Aqil, A., Lippeveld, T. and Hozumi, D. (2009) 'PRISM framework: a paradigm shift for designing, strengthening and evaluating routine health information systems', Health Policy and Planning, 24, pp.217-228.

[13] Watkins, M.D. (2013) What is organizational culture? And Why should we care? Available at: https://hbr.org/2013/05/what-is-organizational-culture(Accessed: 9 February 2020).

[14] Ajzen, I. (2005) Laws of human behavior: symmetry, compatibility, and attitude-behavior correspondence. In: Beauducel A., Biehl B., Bosniak M. et al. (eds). Multivariate Research Strategies. Aachen: Shaker Verlag, 3-19.

[15] Veyrat, P. (2016) Organizational Climate Definition: Everything you need to know. Available at: https://www.heflo.com/blog/hr/organizational-climate-definition/(Accessed: 9 February 2020).

[16] Manya, A. and Nielsen, P. (2016) 'Reporting practices and data quality in health information systems in developing countries: An exploratory case study in Kenya', Journal of Health Informatics in Developing Countries, 10(1), pp.114-126.

[17] Chandler, J. et al. (2017) 'Introduction', in Higgins, J.P.T. et al. (ed.) Cochrane Handbook for Systematic Reviews of Interventions Version 5.2.0 (Updated February 2017). Oxford, UK: UK Cochrane Center, pp.1-50.

[18] MacGill, M. (2019) What is a systematic review in research? Available at: https://www.medicalnewstoday.com/articles/281283.php(Accessed: 16 February 2020). 
Health Informatics - An International Journal (HIIJ) Vol.9, No.4, November 2020

[19] Fineout-Overholt, E. and Johnston, L. (2005) 'Teaching EBP: asking searchable, answerable clinical questions', Worldviews On Evidence-Based Nursing, 2, pp.157-160.

[20] Pluye, P. et al. (2011) Proposal: A mixed methods appraisal tool for systematic mixed studies reviews. Available at:http://mixedmethodsappraisaltoolpublic.pbworks.com (Accessed: 2 February 2020).

[21] Kiwanuka, A., Kimaro, H.C. and Senyoni, W. (2015) 'Analysis of the acceptance process of district health information systems (DHIS) for vertical health programmes: A case study of TB, HIV/AIDS and Malaria programmes in Tanzania', The Electronic Journal of Information Systems in Developing Countries, 70(8), pp.1-14.

[22] Husain, Z., Saikia, N. and Bora, R.S. (2012) ' Opportunities and challenges of health management information system in India, A case study of Uttarakhand', Munich Personal RePEc Archive, 13(57), DOI: https://mpra.ub.uni-muenchen.de/40014.

[23] Askar, A., Ardakani, M. and Mojdzade, R. (2017) 'Bridging the gaps in the health managementinformation systems: a case study from Somaliland, Somalia', Eastern Mediterranean Health Journal, $\quad$ 23(11), pp.764-772.

[24] Asah, F. and Saebo, J.I. (2017) 'Analyzing inhibitors of integrating and routinizing health information systems for universal health coverage: the case of Cameroon', Journal of Health Informatics in Africa, 4(1), pp.114-121.

[25] Astuti, H.M., Herdiyanti, A. and Iriandani, N.(2015) 'Factors influencing the success of hospitalmanagement information systems in a mental hospital in Indonesia', International Journal of Information System and Engineering, 3(1), DOI: 10.24924/ijise/2015.11/v3.iss1/18.26.

[26] Jalghoum, Y. et al. (2019) 'Challenges to healthcare information systems development: The case of Jordan', International Journal of Healthcare Management, DOI: 10.1080/20479700.2019.1658159.

[27] Ahanhanzo, Y.G. et al., (2014) 'Factors associated with data quality in the routine health information system of Benin', Archives of Public Health, 72(25), DOI: http://www.archpublichealth.com/content/72/1/25.

[28] Cheburet, S.K. and Odhiambo-Otieno, G.W. (2016a) 'Process factors influencing data quality of routine health management information system quality: Case of Uasin Gishu County referral hospital, Kenya', International Research Journal of $\quad$ Public and Environmental Health, 3(6), $\quad$ pp.132139.

[29] Cheburet, S.K. and Odhiambo-Otieno, G.W. (2016b) 'Organizational factors affecting data quality of routine health management information system quality: Case of Uasin Gishu County referral hospital, Kenya', International Research Journal of Public and Environmental Health, 3(9), pp.201-208.

[30] Dufera, F.N. et al. (2018) 'Assessment of behavioural and organizational determinants of HMIS performance in Beghi, District West Wollega, Oromia, Ethiopia', Journal of Health, Medicine and Nursing, 46, pp.231-237.

[31] Endriyas, M. et al. (2019) 'Understanding performance data: health management information system data accuracy in Southern Nations Nationalities and People's Region, Ethiopia', BMC Health Services Research, 19(175), DOI: https://doi.org/10.1186/s12913-019-3991-7.

[32] Farzandipur, M., Jeddi, F.R. and Azimi, E. (2016) 'Factors affecting successful implementation of hospital information systems', ACTA Informatica Medica, 24(1), pp.51-55.

[33] Hagan, R.O. et al. (2017) 'National assessment of data quality and associated system-level factors in Malawi', Global Health: Science and Practice, 5(3), pp.367-380.

[34] Harikumar, S.(2012) Evaluation of health management information system- A study of HMIS in Kerala. Thiruvananthapuram, Kerala: Sree Chitra Tirunal Institute for Medical Sciences and Technology.

[35] Kagoya, H.R. and Kibuule, D. (2018) 'Quality assurance of health management information system in Kayunga district, Uganda', African Evaluation Journal, 6(2), a238. https://doi.org/10.4102/aej.v6i2.238.

[36] Kasambara, A. et al. (2017) 'Assessment of implementation of the health management information system at the district level in Southern Malawi', Malawi Medical Journal, 29(3), pp.240-246.

[37] Kumwenda, B. et al. (2014) 'Factors affecting data quality in the Malawian health management information system', Health Informatics, 1(28), 286-292. DOI: 10.2316/P.2014.815-028.

[38] Malik, I.H. and Hameed, S. (2012) ' Factors affecting implementation of hospital management information systems in Pakistan', International Journal of Physical Sciences, 7(20), pp.2818-2828.

[39] Mishra, A. et al., 2012 ' Determinants of health management information systems performance: 
Health Informatics - An International Journal (HIIJ) Vol.9, No.4, November 2020

Lessons from a district level assessment', BMC Proceedings, 6(Suppl 5), Doi:10.1186/1753-6561-6S5- O17.

[40] Simba, D.O. and Mwangu, M.A. (2006) 'Factors influencing quality of health management information system (HMIS) data, the case of Kinondoni district in Dae Es Salaam region, Tanzania', East African Journal of Public Health, 3(1), pp.28-31.

[41] Tadesse, K., Gebeye, E. and Tadesse, G. (2014) 'Assessment of health management information system implementation in Ayder referral hospital, Mekelle, Ethiopia', International Journal of Intelligent Information Systems, 3(4), pp.34-39.

[42] Teklegiorgis, K. et al. (2016) 'Level of data quality from health management information systems in a resource limited setting and its associated factors, eastern Ethiopia', South African Journal of Information Management, 17(1), DOI: http://dx.doi.org/10.4102/sajim.v17i1.612

[43] Wagenaar, B.H. et al. (2015) 'Effects of a health information system data quality intervention on concordance in Mozambique: time-series analyses from 2009-2012', Population Health Metrics, 13(9), DOI: 10.1186/s12963-015-0043-3.

[44] Ahmadian, L. et al. (2017) 'Challenges of using hospital information systems by nurses: comparing academic and non-academic hospitals', Electronic Physician, 9(6), pp.4625-4630.

[45] Kpobi, L., Swartz, L. and Ofori-Atta, A.L. (2018) 'Challenges in the use of mental health information system in a resource-limited setting: Lessons from Ghana', BMC Health Services Research, 18(98), DOI 10.1186/s12913-018-2887-2.

[46] Nyamtema, A.S. (2010) 'Bringing the gaps in the health management information system in the context of a changing health sector', BMC Medical Informatics and Decision Making, 10(36), DOI: $\quad$ http://www.biomedcentral.com/1472-6947/10/36

[47] Soontornpipit, P. et al. (2016) 'The study of hospital information system in the $8^{\text {th }}$ health region', Procedia Computer Science, 86, pp.252-256.

[48] Wilms, M.C. et al., (2014) 'An in-depth, exploratory assessment of the implementation of the national health information system at a district level hospital in Tanzania', BMC Health Services Research, 14(91), DOI: http://www.biomedcentral.com/1472-6963/14/91

[49] Ismail, A. et al., (2012) ' The implementation of hospital information system (HIS) in tertiary hospitals in Malaysia: a qualitative study', Malaysian Journal of Public Health Medicine, 10(2), pp.16-24.

[50] Ledikwe, J.H. et al. (2014) 'Improving the quality of health information: a qualitative assessment of data management and reporting systems in Botswana', Health Research Policy and Systems, 12(7), DOI: http://www.health-policy-systems.com/content/12/1/7

[51] Mpofu, M. et al. (2014) 'Strengthening monitoring and evaluation ( $\mathrm{M} \&$ E) and building sustainablehealth information systems in resource limited countries: lessons learned from an M\&E task-shifting initiative in Botswana', BMC Public Health, 14(1032), DOI: http://www.biomedcentral.com/1471- 2458/14/1032.

[52] Wandera, S.O. et al. (2019) 'Facilitators, best practices and barriers to integrating family planning data in Uganda's health management information system', BMC Health Services Research, 19(327), https://doi.org/10.1186/s12913-4151-9.

[53] Dehury, R.K. and Chatterjee, S.C. (2018) 'Assessment of health management information system for monitoring of maternal health in Jaleswar Block of Balasore District, Odisha, India', Indian Journal of Public Health, 62(4), pp.259-264.

[54] Moucheraud, C. et al. (2017) 'Sustainability of health information systems: a three-country qualitativestudy in southern Africa', BMC Health Services Research, 17(23), DOI: 10.1186/s12913016-1971-8.

[55] Park, Y.T. and Lee, J. (2014) 'Factors affecting electronic medical record system adoption in small Korean hospitals', Healthcare Informatics Research, 20(3), pp.183-190.

[56] Garrib, A. et al. (2008) 'An evaluation of the district health information system in rural South Africa', South African Medical Journal, 98(7), pp.549-552.

[57] Kuyo, R.O., Muiruri, L. and Njuguna, S. (2018) 'Organizational factors influencing the adoption of the district health information system 2 in Uasin Gishu County, Kenya', International Journal of Medical Research, 7(10), pp.48-57.

[58] Akhlaq, A. et al., (2016) 'Barriers and facilitators to health information exchange in low- and middleincome country settings: a systematic review', Health Policy and Planning, 31, pp.1310-1325.

[59] Mohamadali, N.A. and Aziz, N.F.A. (2017a) 'The organizational factors as barriers for sustainable health information systems (HIS)- A review', Procedia Computer Science, 124, pp.354-361. 
[60] Mohamadali, N.A. and Aziz, N.F.A. (2017b) 'The technology factors as barriers for sustainable health information systems (HIS)- A review', Procedia Computer Science, 124, pp.370-378.

[61] Garavand, A. et al. (2016)'Factors influencing the adoption of health information technologies: a systematic review', Electronic Physician, 8(8), pp.2713-2718.

[62] Ross, J. et al. (2016)'Factors that influence the implementation of e-health: A systematic review of systematic reviews', Implementation Science, 11(146), DOI :10.1186/s13012-016-0510-7.

[63] Sezgin, E and Yildirim, S.O. (2014) 'A literature review on attitudes of health professionals towards health information systems: From e-health to m-health', Procedia Technology, 16, pp.1317-1326.

[64] Lua, F. et al. (2010) 'A review on systematic reviews of health information system studies', Journal of the American Medical Informatics Association, 17, pp.637-645.

[65] Shiferaw, A.M. et al. (2017) 'Routine health information system utilization and factors associated thereof among health workers at government health institutions in East Gojjam Zone, Northwest Ethiopia', BMC Medical Informatics and Decision Making, 17(116), DOI 10.1186/s12911-0170509- 2.

[66] Chandler, C.I.R. et al. (2009) 'Motivation, money and respect: A mixed method study of Tanzania non-physician clinicians, Social Science Medicine, 68, pp.2078-2088.

[67] Dambisya, Y.M. and Matinhure, S. (2012) 'Policy and programmatic implications of task shifting in Uganda: A case study', BMC Health Services Research, 12(61), pp.1-10.

[68] Lehmann, U. et al. (2009) 'Task shifting: the answer to the human resources crisis in Africa?', Human Resource Health, 7(49), https://doi.org/10.1186/1478-4491-7-49.

[69] Braa, J., Heywood, A. and Sahay, S. (2012) 'Improving quality and use of data through data-use workshops: Zanzibar, United Republic of Tanzania', Bulletin of the World Health Organization, 90(5), pp.379-384.

[70] Watkins, M.D. (2013) What is organizational culture? And Why should we care? Available at: https://hbr.org/2013/05/what-is-organizational-culture(Accessed: 9 February 2020).

[71] Clarke, A. et al. (2015) 'Implementing electronic patient record systems (EPRs) into England's acute, mental health and community care trusts: A mixed methods study', BMC Medical Informatics and Decision Making, 15(85), DOI: 10.1186/s12911- 015-0204-0.

[72] Gopalan, S.S. et al. (2013) 'Health sector demand-side financial incentives in low- and middleincome countries: A systematic review on demand- and supply-side effects', Social Science Medicine, 21, pp.40-48.

[73] Grammatikopoulos, I.A. et al. (2013) 'Job motivation factors and performance incentives as efficient management tools: A study among mental health professionals', Archives of Hellenic Medicine, 30, pp.46-58.

[74] Helen, T. (2011) Assessment of the health management information system implementation status in public health facilities in Bahir-Dar city. Master's thesis, School of Information Science, Addis Ababa University.

[75] Khajouei, R. et al. (2011) 'Clinicians satisfaction with CPOE ease of use and effect on clinicians' workflow, efficiency and medication safety', International Journal of Medical Information, 80(5), pp.297-309.

[76] Mdege, N.D., Chindove, S. and Ali, S. (2013) 'The effectiveness and cost implications of taskshifting in the delivery of antiretroviral therapy to HIV-infected patients: A systematic review', Health Policy Plan, 28(3), pp.223-236.

[77] Admon, A.J. et al. (2013) 'Assessing and improving data quality from community health workers: a successful intervention in Neno, Malawi;, Public Health Action, 3(1), pp.56-59.

[78] Chaulagai, C.N. et al. (2005) 'Design and implementation of a health management information system in Malawi: issues, innovations and results', Health Policy Plan, 20(6), pp.375-384.

[79] Huaman, M.A. et al. (2009) 'Impact of two interventions on timeliness and data quality of an electronic disease surveillance system in a resource limited setting (Peru): a prospective evaluation', BMC Medical Informatics and Decision Making, 9(16),DOI: 10.1186/1472-6947-9-16.

[80] Makombe, S.D. et al. (2008) 'Assessing the quality of data aggregated by antiretroviral treatment clinics in Malawi', Bulletin of the World Health Organization, 86(4), pp.310-314.

[81] Mate, K.S. et al. (2009) 'Challenges for routine health system data management in a large public programme to prevent mother-to-child HIV transmission in South Africa, PLoS ONE, 4(5): e5483. https://doi.org/10.1371/journal.pone.0005483

[82] Mphatswe, W. et al. (2012) 'Improving public health information: a data quality intervention in KwaZulu-Natal, South Africa', Bulletin of the World Health Organization, 90(3), pp.176-182. 